\title{
Targeting the organ-specific adiposity
}

\author{
Gianluca lacobellis ${ }^{1}\left[\right.$ Giuseppe Barbaro $^{2}$
}

Received: 13 July 2018 / Accepted: 23 July 2018 / Published online: 14 August 2018

(c) Springer Nature Switzerland AG 2018

The organ-specific adiposity is the topic that Manno and colleagues sought to address in their paper published in Eating and Weight Disorders [1]. The authors evaluated ultrasound-measured different fat depots in overweight and obese subjects and suggested that peri- and para-renal fat may have more systemic effects than epicardial fat to which they attributed a more likely local influence and control of the heart. Particularly, Manno found that both perirenal and pararenal fat depots were better correlated with a surrogate index of insulin resistance whereas epicardial fat was associated with LDL-cholesterol [1]. This is certainly a subject deserving an in-depth analysis.

In the past few years, the perception and approach to obesity have changed profoundly. The focus rapidly narrowed from the wider picture of obesity to the visceral abdominal fat and eventually to the intra-cellular fat infiltration. It is not a matter of terminology, but rather of contiguity and specificity. Fat cells are not all the same, and they do have functions and effects depending on the organ they surround and, under pathological circumstances, infiltrate and ultimately damage. Adipose tissue gene and adipokines expression is specific for the function of the contiguous organ. Fat becomes detrimental not only when excessive and then inappropriately ectopic, but also when the physiological transcriptome and proteome are downregulated [2]. The upregulation of the adipose tissue pro-inflammatory and pro-atherogenic genes is reciprocally regulated by the metabolic and hemodynamic milieu of the adjacent organ. Hence, the vicinity and specificity are key aspects of the cross-talk between the fat and its organ.

Gianluca Iacobellis

giacobellis@med.miami.edu

1 Division of Diabetes, Endocrinology and Metabolism, Department of Medicine, University of Miami, Miller School of Medicine, 1400 NW 10th Ave, Dominion Tower suite 805-807, Miami, FL 33136, USA

2 Department of Experimental Medicine, Section of Medical Pathophysiology, University of Rome Sapienza, Rome, Italy
The paper by Manno and colleagues provides us with the opportunity to briefly discuss the anatomy of these fat depots and highlight a remarkable difference. Epicardial adipose tissue is a peculiar fat depot with unique embryological, anatomical, genetic and biomolecular properties [3]. Speaking only about anatomy, epicardial fat is in direct physical contiguity to the myocardium, as it lies between the myocardium and the visceral layer of the pericardium and no muscle fascia separates the two tissues that actually share the same microcirculation. On the contrary, perirenal adipose tissue is the layer of fat adjacent to the renal capsule, a fibrous tissue enclosing the renal parenchyma. Pararenal fat lies even more externally, outside the renal fascia. Hence, epicardial fat is unique and different from any other visceral fat depots, as there is no anatomical barrier that separates it from the heart. Whether this implies that epicardial fat may exert more local than systemic effects is likely correct, although some studies suggested a correlation between epicardial fat adipokines expression and intracoronary circulating levels [4]. Epicardial fat is indeed differently distributed within the heart and can be found within the myocardium, around the coronary arteries, ventricles and atria [3]. Different epicardial fat depots can have specific functions and genetic profile and therefore different clinical implications. Perirenal fat has some analogies with the pericardial fat, the depot located between the visceral and the parietal layer of the pericardium, so externally to the myocardium [5]. Whilst pericardial fat has been largely associated with increased cardiovascular risk, the role of perirenal fat depot has not been well-established, yet. The paper by Manno and colleagues suggested a relationship of perirenal fat with insulin resistance, although this should be better evaluated with the euglycemic insulin clamp [6]. It would be interesting to look at the potential interaction between the perirenal and periadrenal fat depot, as the latter was found to be metabolically active [7].

Notably, the article by Manno confirmed how these organspecific fat depots, such as the epicardial fat, can be easily detected and measured with readily available and non-invasive ultrasound methods [8]. Echocardiographic-measured 
epicardial fat thickness has been widely reported as a good predictor of cardiovascular risk and therapeutic target of pharmaceutical agents modulating the fat [9]. Ultrasound assessment of organ fat depots should be implemented as routine diagnostic procedure to better stratify the cardiometabolic risk. Future directions would be aimed to pharmaceutically modulate organ-specific fat depots transcriptome to ultimately benefit the target organs.

\section{Compliance with ethical standards}

Conflict of interest The authors declare that there is no competing interest.

Ethical approval This article does not contain any studies with human participants performed by any of the authors.

Informed consent For this type of study informed consent is not required.

\section{References}

1. Manno C, Campobasso N, Nardecchia A, Triggiani V, Zupo R, Gesualdo L, Silvestris F, De Pergola G (2018) Relationship of para- and perirenal fat and epicardial fat with metabolic parameters in overweight and obese subjects. Eat Weight Disord. https ://doi.org/10.1007/s40519-018-0532-z (Epub ahead of print)

2. McAninch EA, Fonseca TL, Poggioli R, Panos AL, Salerno TA, Deng Y, Li Y, Bianco AC, Iacobellis G (2015) Epicardial adipose tissue has a unique transcriptome modified in severe coronary artery disease. Obesity 23:1267-1278

3. Iacobellis $\mathrm{G}$ (2015) Local and systemic effects of the multifaceted epicardial adipose tissue depot. Nat Rev Endocrinol 11:363-371

4. Iacobellis G, di Gioia CR, Cotesta D, Petramala L, Travaglini C, De Santis V, Vitale D, Tritapepe L (2009) Letizia C Epicardial adipose tissue adiponectin expression is related to intracoronary adiponectin levels. Horm Metab Res 41:227-223

5. Iacobellis G (2009) Epicardial and pericardial fat: close, but very different. Obesity 17:625

6. Iacobellis G, Leonetti F (2005) Epicardial adipose tissue and insulin resistance in obese subjects. J Clin Endocrinol Metab 90:6300-6302

7. Letizia C, Petramala L, Di Gioia CR, Chiappetta C, Zinnamosca L, Marinelli C, Iannucci G, Ciardi A, De Toma G, Iacobellis G (2015) Leptin and adiponectin mRNA expression from the adipose tissue surrounding the adrenal neoplasia. J Clin Endocrinol Metab 100:E101-E104

8. Iacobellis G, Willens HJ (2009) Echocardiographic Epicardial Fat: A Review of Research and Clinical Applications. J Am Soc Echocardiogr 22:1311-1319

9. Iacobellis G, Mohseni M, Bianco SD, Banga PK (2017) Liraglutide causes large and rapid epicardial fat reduction. Obesity 25:311-316 